\title{
Embolia pulmonar en presencia de infección por SARS CoV-2: una presentación atípica en un paciente joven
}

\author{
Pulmonary embolism in the presence of SARS CoV-2 \\ infection: An atypical presentation in a young patient
}

\author{
Maria Cristina Martínez Ávila ${ }^{1}$, Amilkar José Almanza Hurtado ${ }^{2}$, Zulay Mondol ${ }^{3}$, \\ Alvano Trespalacios Sierra ${ }^{4}$, Alejandro De Jesús Blanquicett ${ }^{5}$ Mario Andrés Pineda \\ Paternina $^{6}$, Kelly Mesa Gamarra ${ }^{7}$
}

\section{Resumen}

INTRODUCCIÓN: Las infecciones virales como la emergente por el COVID-19 producen estados de hipercoagulabilidad y proinflamatorios, que pueden desencadenar tromboembolismo pulmonar y representan un reto diagnóstico y terapéutico para el personal de salud. Debido a que es una enfermedad nueva, aún no se conocen la totalidad de sus síntomas. Se presenta el caso clínico de un paciente con disnea aguda y hemoptisis secundario a un tromboembolismo pulmonar segmentario, en contexto de infección confirmada por COVID-19.

PRESENTACIÓN DE CASO: paciente masculino de 37 años con antecedentes de hipertensión arterial, sin factores de riesgo protrombóticos, con cuadro agudo de dolor torácico, disnea y hemoptisis franca. Paraclínicos con dímero D y ferritina elevada, prueba de PCR para SARS CoV-2 positiva, angiotomografía de tórax con evidencia de tromboembolismo pulmonar segmentario y patrón de vidrio esmerilado en segmento basal anterior del lóbulo inferior. Cumplió manejo antibiótico y anticoagulante con enoxaparina a dosis plena y posteriomente, con anticoagulante oral directo y rivaroxaban de manera ambulatoria.

CONCLUSIÓN: La infección por el nuevo coronavirus puede desencadenar eventos trombóticos como embolismo pulmonar, que se convierten en un reto diagnóstico y terapéutico para los clínicos, con presentación que va desde síntomas generales hasta más específicos, entre los que destaca la hemoptisis, razón por la cual, el presente artículo tiene como objetivo mostrar la importancia de una evaluación secuencial al momento de realizar el diagnóstico clínico. De igual forma, se expone la necesidad de no descuidar
1 Médica Epidemióloga. Unidad de Cuidados Intensivos Nuevo Hospital Bocagrande, Cartagena, Colombia.

2 Médico Internista. Universidad del Sinú, sede Cartagena. Unidad de Cuidados Intensivos Nuevo Hospital Bocagrande. Cartagena, Colombia.

${ }^{3}$ Médica, Residente de Medicina Interna. Universidad del Sinú, sede Cartagena, Colombia.

4 Médico General Unidad de Cuidados Intensivos Nuevo Hospital Bocagrande, Cartagena, Colombia.

5 Médico General, Corporación Universitaria Rafael Núñez. Cartagena, Colombia.

${ }^{6}$ Médico, Residente de Medicina Interna, Universidad del Sinú, sede Cartagena, Colombia.

${ }^{7}$ Médica General, Universidad de Cartagena. Cartagena, Colombia.

Autor de Correspondencia María Cristina Martínez Ávila Correo electrónico:

cristina.martinezavila@gmail.com

Recibido: 24/09/2020

Aceptado: 15/01/2021 
las manifestaciones atípicas relacionadas con estados de hipercoagulabilidad, ya que pueden sugerir patologías que ponen en riesgo la vida del paciente.

Palabras clave: Hemoptisis; tromboembolismo pulmonar; hipercoagulabilidad; COVID-19; SARS CoV-2; presentación atípica.

\begin{abstract}
INTRODUCTION: Viral infections such as the one emerging from COVID 19 produce hypercoagulable and pro-inflammatory states that can trigger pulmonary thromboembolism, being a diagnostic and therapeutic challenge for health personnel. Being a new disease, all of its symptoms are not yet known. We present the clinical case of a patient with acute dyspnea and hemoptysis secondary to segmental pulmonary embolism, in the context of confirmed COVID-19 infection.

CASE PRESENTATION: A 37-year-old male patient with a history of arterial hypertension, without prothrombotic risk factors, with acute symptoms of chest pain, dyspnea and frank hemoptysis. Paraclinical patients with d-dimer and elevated ferritin, positive SARSCOV2 RTPCR, chest CT angiography showing segmental pulmonary thromboembolism and ground glass pattern in the anterior basal segment of the lower lobe. He complied with antibiotic and anticoagulant management with full dose enoxaparin and subsequently with direct oral anticoagulant, rivaroxaban on an outpatient basis.

CONCLUSION: Infection by the new coronavirus can trigger thrombotic events such as pulmonary embolism, which becomes a diagnostic and therapeutic challenge for clinicians, with a presentation that ranges from general to more specific symptoms, among which hemoptysis stands out, which is why which, the present one aims to show the importance of a sequential evaluation when making the clinical diagnosis. In the same way, to expose the need not to neglect the atypical manifestations related to hypercoagulable states, since they cannot suggest pathologies that put the patient's life at risk.
\end{abstract}

Keywords: Hemoptysis; pulmonary embolism; hypercoagulability; COVID 19; SARS CoV-2; atypical presentations.

\section{Introducción}

En diciembre de 2019 apareció una neumonía de origen desconocido en Wuhan, provincia de Hubei, China, que se fue extendiendo por todo el mundo. El 9 de enero de 2020, el Centro para el Control y la Prevención de Enfermedades de China informó que el agente causante de la infección era un nuevo coronavirus 2 del síndrome respiratorio agudo grave (SARSCoV-2) o COVID-19, clasificado así por la Organización Mundial de la Salud (OMS) $(1,2)$.

Los síntomas respiratorios de COVID-19 son extremadamente heterogéneos, van desde síntomas mínimos hasta hipoxia significativa con síndrome de distrés respiratorio agudo (SDRA), que pueden conducir a la muerte. Pueden encontrarse también signos y síntomas inespecíficos para cualquier enfermedad, como fiebre, tos seca, fatiga, mialgia y disnea y otros menos comunes como cefalea, mareos, náuseas, do- lor abdominal, vómitos y diarrea $(3,4)$. Los síntomas respiratorios en la COVID-19, están mediados por un estado proinflamatorio y de hipercoagulabilidad, que se evidencia con niveles séricos elevados de lactato deshidrogenasa, ferritina, proteína $\mathrm{C}$ reactiva, dímero D e interleucina (5-7). Dichos hallazgos son predisponentes para el desarrollo de una infección viral grave y aumentan el riesgo de complicaciones tromboembólicas en pacientes con COVID-19, en comparación con otras enfermedades sistémicas.

Los perfiles de hipercoagulabilidad observados están relacionados con la disfunción endotelial, siendo este un factor importante en la regulación trombótica vinculado a una mayor producción de trombina y reducción de la fibrinólisis. Esto, a su vez, induce el aumento de la permeabilidad microvascular e invasión viral (8-10), dando origen a presentaciones atípicas a nivel radiológico y clínico (por ejemplo, la hemoptisis) en pacientes con COVID-19 (11). 
Se presenta el caso clínico de un paciente con hemoptisis como manifestación inicial de infección respiratoria aguda por COVID-19, con posterior desarrollo de tromboembolismo pulmonar (TEP).

\section{Reporte de caso}

Paciente masculino de 37 años con antecedente de hipertensión arterial, manejado con un bloqueador de los receptores de angiotensina II, quien consulta a hospital en la ciudad de Cartagena, Colombia por cuadro de dolor torácico de instauración aguda localizado en región escapular derecha, de intensidad 8/10 según escala análoga de dolor, de carácter lancinante, asociado a hemoptisis franca. Niega síntomas respiratorios, disnea y picos febriles; niega viajes recientes o contacto directo con personas sospechosas o positivas para COVID-19.

A su ingreso se encuentra afebril (36.4 Co), taquicárdico (110 latidos por minuto), taquipneico (28 respiraciones por minuto), saturación de $95 \%$ con fracción inspirada de oxígeno de $21 \%$, con disnea leve, estertores ocasionales en la base derecha; el resto del examen sin hallazgos significativos. Se realiza radiografía de tórax en la cual se evidencia opacidad basal en pulmón derecho, cardiomegalia grado I. Electrocardiograma con taquicardia sinusal, patrón de ritmo S1Q3T3 (Figura 1). Perfil hematológico con reactantes de fase aguda aumentados, proteína $C$ reactiva 24 , leucocitos 22400, 89 \% neutrófilos, deshidrogenasa láctica 293 y en los gases arteriales hipoxemia leve $\mathrm{PaO} 2 / \mathrm{FiO} 2$ de 293 (presión arterial de oxígeno/fracción inspirada de oxígeno).

Se hospitaliza en sala general con aislamiento respiratorio, bajo diagnóstico de infección respiratoria aguda versus tuberculosis pulmonar, sin descartar otras causas de hemoptisis como neoplasia o absceso pulmonar. Se inicia manejo antibiótico de amplio espectro con betalactámicos y macrólidos, para cubrimiento de gérmenes atípicos y se solicitan paraclínicos de extensión para confirmación diagnóstica, estratificación de riesgo y pronóstico. La tomografía computarizada de alta resolución del tórax mostró opacidad no homogénea con área de vidrio deslustrado en el segmento basal anterior del lóbulo inferior derecho con áreas

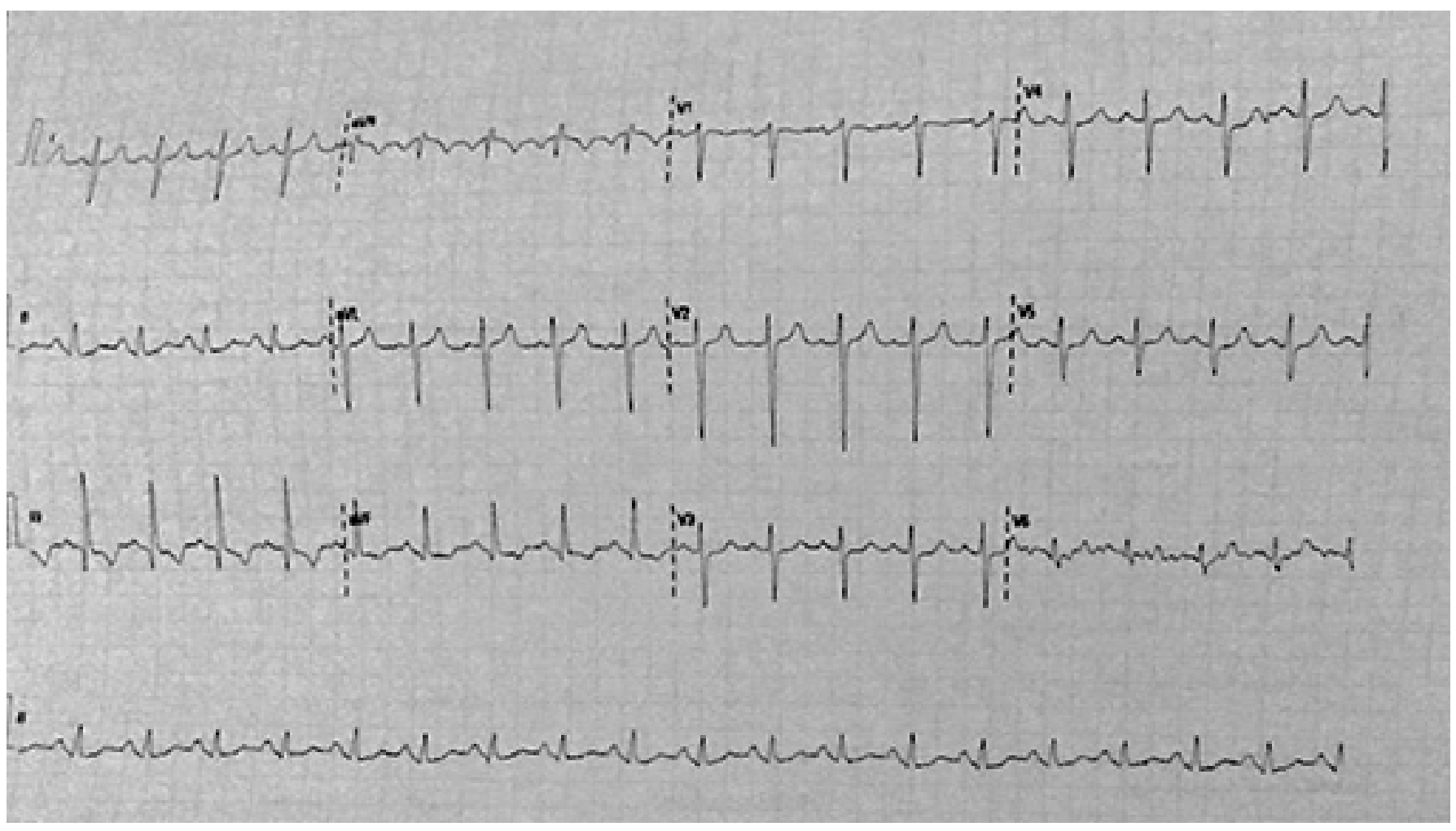

Figura 1. Electrocardiograma con patrón S1Q3T3 
hipodensas en su interior de bordes irregulares, difuminados de aspecto inflamatorio y atelectasias subsegmentarias que se extienden hasta la pleura (Figura 2).

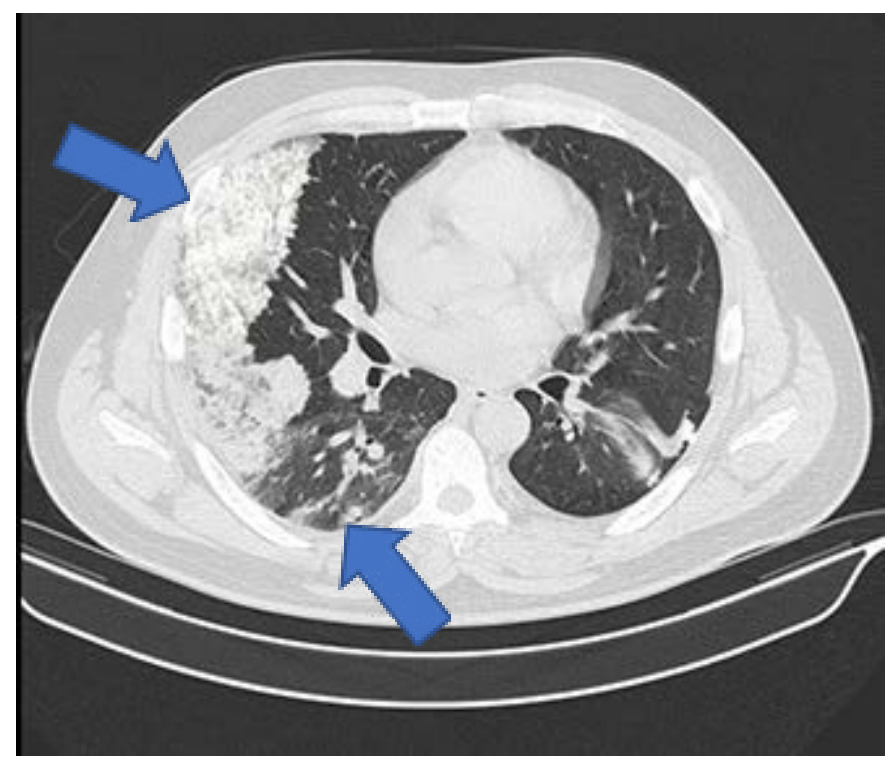

Figura 2. Tomografía de tórax. Opacidad heterogénea con vidrio esmerilado irregular y con atelectasias a nivel de pulmón derecho.

En contexto de la pandemia actual, se solicita hisopado para prueba de PCR para SARS CoV-2 la cual resulta positiva; baciloscopias negativas, dimero D en 5860 y ferritina en 2090. Dada la persistencia de taquicardia, taquipnea y hemoptisis, en conjunto con los hallazgos electrocardiográficos y factores protrombóticos elevados, es trasladado a unidad de cuidado crítico de COVID-19 y se solicita angiotomografía de tórax (Figura 3). En esta se evidencia tromboembolismo pulmonar, con compromiso de la arteria lobar inferior derecha, segmentaria anterior, segmentaria basal inferior y basal medial, con cambios parenquimatosos secundarios al tromboembolismo, dado por infarto pulmonar periférico y atelectasias confluentes en el resto del lóbulo inferior derecho, por lo que se adiciona al manejo, enoxaparina a dosis plena.

Otras pruebas bioquímicas, enzimas cardiacas, función renal y hepática, electrolitos y parámetros de coagulación, fueron normales. También se descartaron condiciones trombofílicas, deficiencia de antitrombina, proteína $\mathrm{S}$ y $\mathrm{C}$, factor $\mathrm{V}$ Leiden, variantes de protrombina y anticuerpos antifosfolípidos. El ecocardiograma transtorácico mostró un tamaño normal del ventrículo derecho, sin evidencia de presiones elevadas de la arteria pulmonar u otras anomalías y el doppler venoso de miembros inferiores, fue negativo para trombosis venosa profunda.

El paciente presenta evolución satisfactoria, completando esquema antibiótico y anticoagulación terapéu-

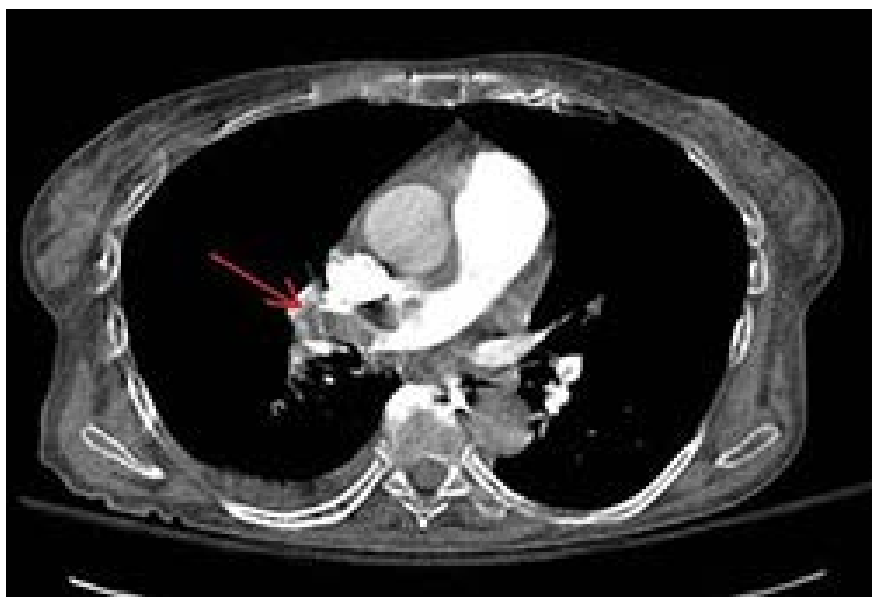

Figura 3. Angiotomografía de tórax

tica con heparina de bajo peso molecular, sin disnea, ni datos activos de respuesta inflamatoria sistémica. Se considera dar alta médica con manejo ambulatorio con anticoagulantes orales directos (DOACs), inhibidor del factor Xa (rivaroxabán) $50 \mathrm{mg}$ por vía oral cada 12 horas y seguimiento ambulatorio.

\section{Discusión y conclusiones}

Los síntomas respiratorios de COVID-19 son extremadamente heterogéneos y van desde mínimos, hasta una hipoxia significativa con síndrome de distrés respiratorio agudo (SDRA), que pueden conducir a la muerte (3). A medida que se conoce un poco más sobre esta patología, se identifican de forma precoz las presentaciones atípicas asociadas a estados de hipercoagulabilidad (12). Por ello se trae a colación el reporte de caso de un paciente joven con antecedente de hipertensión arterial, sin factores de riesgo ni antecedentes protrombóticos, atendido en un hospital de mediana complejidad y diagnosticado con tromboembolismo pulmonar, secundario a infección por COVID-19 con tratamiento médico exitoso.

La hemoptisis es la principal manifestación en la 
evolución, hasta debutar con un cuadro de disnea, asociado a otros síntomas respiratorios e infecciosos. La hemoptisis no es un síntoma típico de presentación de COVID-19. Estudios a nivel mundial no han reportado casos o describen tasas extremadamente bajas: aproximadamente el $1 \%$ de los pacientes en China, alcanzando hasta el $5 \%$ en Europa y América (1315). Hasta el momento no hay ningún caso reportado en Colombia, por lo tanto, no ha sido incluido en los Lineamientos y Manejo de COVID-19 del Ministerio de Salud y Protección Social.

A pesar de que el mecanismo y causa exacta de la hemoptisis aun no son claros $(11,12)$, su incidencia se vuelve relativamente mayor, alcanzando hasta el $13 \%$ en pacientes con émbolos pulmonares $(16,17)$. Cabe resaltar que en pacientes con neumonía viral por COVID-19, los síntomas respiratorios pueden empeorar rápidamente. Incluso es posible la aparición de insuficiencia respiratoria aguda hipóxica, debido a la progresión de la enfermedad en asociación a los cambios fisiopatológicos y estado protrombótico secundario; todos estos pueden ser también atribuibles a un TEP no diagnosticado. Se confirma así, el papel de las infecciones graves como precipitantes del tromboembolismo venoso y la asociación entre el SARS CoV2, la hipercoagulabilidad y la lesión pulmonar aguda $(18,19)$.

Conociendo el estado protrombótico e inflamatorio debido a la infección, las guías recomiendan la tromboprofilaxis con heparinas de bajo peso molecular subcutánea dos veces al día, en dosis profilácticas o intermedias (7-9). Estas consideraciones se ajustan a las características y condiciones de cada paciente. En el caso clínico expuesto, inicialmente no se dio tromboprofilaxis por la hemoptisis; tras el diagnóstico de TEP se indicó anticoagulación a dosis terapéuticas y al egreso hospitalario con DOACs por un período de tres meses, en aras de disminuir recurrencia de eventos trombóticos, reconociendo que cuentan con un mejor perfil de seguridad y adherencia en el paciente $(20,21)$. En relación con los hallazgos imagenológicos, de forma habitual está descrito en la clasificación de CO-RADS (COVID-Reporting and Data System classification) evidencia de vidrio esmerilado, patrón deslustrado, consolidación de las regiones subpleurales o periféricas bilaterales (22-23). No es fácil diagnosticar COVID-19 con una imagen única de distribución aislada y heterogénea, como la del caso que se presenta, quien además su único síntoma era la hemoptisis y taquipnea, sin antecedentes de contacto con pacientes positivos o sospechosos. Por tal razón, se consideraron otros diagnósticos diferenciales al ingreso. Una vez diagnosticada la infección por SARS CoV2, el deterioro de la mecánica respiratoria sin hipoxia, con taquicardia sinusal asociado a patrón S1Q3T3, fue lo que llevó a la realización de la angioescanografía como prueba gold estándar para el diagnóstico definitivo de tromboembolismo pulmonar. En el paciente se realizaron estudios sin identificar causas predisponentes para el TEP, lo que indicaría que fue una complicación de la infección por COVID-19 $(19,22)$.

$\mathrm{El}$ tromboembolismo pulmonar per se, exige un reto diagnóstico y terapéutico para evitar desenlaces fatales. Como manifestación atípica del COVID-19, se debe tener presente como una opción diagnóstica para inicio de terapia oportuna. En la práctica clínica debemos estar atentos a las presentaciones inusuales inducidas por estados de hipercoagulabilidad, sus presentaciones pulmonares y extrapulmonares. Dado que la COVID-19 es un estado de hipercoagulabilidad, se hace necesario considerar el tromboembolismo pulmonar como una complicación frecuente y debe sospecharse en cualquier paciente, aun en ausencia de datos clínicos y paraclínicos que sugieran esta patología. Ante la duda diagnóstica, las imágenes deben jugar un papel fundamental. No se debe retrasar el inicio de profilaxis y terapia anticoagulante adecuada y oportuna individualizando cada paciente $(20,21)$.

Finalmente, se considera que el caso descrito demuestra la complejidad de la patología causante de la pandemia actual, donde no se encontró cerco epidemiológico. Adicionalmente, el desconocimiento de las manifestaciones atípicas puede conducir a diagnósticos errados y tardíos, dando lugar a complicaciones o progresión de la enfermedad.

\section{Conflictos de interés}

Los autores declaran no presentar ningún conflicto de interés.

\section{Referencias}

1. Phelan AL, Katz R, Gostin LO. The novel coronavirus originating in Wuhan, China: challenges for global health governance. JAMA. 2020;323(8):709-710 doi:10.1001/ jama.2020.1097

2. Zhou P, Yang X-L, Wang X-G, Hu B, Zhang L, 
Zhang W, et al. A pneumonia outbreak associated with a new coronavirus of probable, bat origin. Nature 2020;579:270-273. doi: 10.1038/s41586020-2012-7

3. Huang C, Wang Y, Li X, et al. Clinical features of patients infected with 2019 novel coronavirus in Wuhan, China. The Lancet. 2020; 395(10223):497 $\square$ 506. doi: 10.1016/S01406736(20)30183-5

4. Wang D, Hu B, Hu C, et al. Clinical characteristics of 138 hospitalized patients with 2019 novel coronavirus-infected pneumonia in Wuhan, China. JAMA. 2020;323(11):1061 $\square$ 1069. doi:10.1001/ jama.2020.1585

5. Chen G, Wu D, Guo W, Cao Y, Huang D, et al. Clinical and immunologic features in severe and moderate coronavirus disease 2019. J Clin Invest 2020;130(5):2620-2629. doi: 10.1172/JCI137244.

6. Han H, Yang L, Liu R, Liu F, Wu KL, Li J, et al. Prominent changes in blood coagulation of patients with SARS-CoV-2 infection. Clin Chem Lab Med (CCLM). 2020;25;58(7):1116-1120. doi: 10.1515/cclm-2020-0188.

7. Lu, Guoguang \& Wang, Jing. (2020). Dynamic changes in routine blood parameters of a severe COVID-19 case. Clinica Chimica Acta. 508. 2020;508:98-102. doi: 10.1016/j.cca.2020.04.034.

8. Spiezia L, Boscolo A, Poletto F, Cerruti L, Tiberio I, Campello E, et al (2020) COVID-19 -related severe hypercoagulability in patients admitted to Intensive Care Unit for acute respiratory failure. Thromb Haemost. 2020;120(6):998-1000. doi: 10.1055/s-0040-1710018

9. Levi M, Van der Poll T. Coagulation and sepsis. Thromb Res. 2017;149:38-44. doi: 10.1016/j. thromres.2016.11.007.

10. Wang M., Hao H., Leeper N.J., Zhu L., Early Career C. Thrombotic regulation from the endothelial cell perspectives. Journal. 2018;38:e90-e95. doi: 10.1161/ATVBAHA.118.310367

11. Edirappuli SD, Venkatesh A. Atypical symptoms in COVID-19: the many guises of a common culprit. BMJ 2020;369:m1375. http://www.bmj. com/content/369/bmj.m1375/rr-12.

12. Abobaker A, Raba A, Aboubaker A. (2020). Extrapulmonary and atypical clinical presentations of COVID $\square$ 19. Journal of Medical Virology.10.1002/jmv.26157. doi: 10.1002/jmv.26157

13. Casey K, Iteen A, Nicolini R, Auten J. COVID-19 pneumonia with hemoptysis: acute segmental pulmonary emboli associated with novel coronavi- rus infection. Am J Emerg Med. 2020;38(7):1544. e1-1544.e3. doi: 10.1016/j.ajem.2020.04.011

14. Joshi S, Bhatia A, Tayal N, Nair D. Hemoptysis as an Initial Presentation of COVID-19 - An Observation. The Journal of the Association of Physicians of India. 2020;68(6):68.

15. Shi F, Yu Q, Huang W, Tan C. 2019 novel coronavirus (COVID-19) pneumonia with hemoptysis as the initial symptom: CT and clinical features. Korean J Radiol 2020;21:537-540

16. Stein PD, Terrin ML, Hales CA, Palevsky HI, Saltzman HA, et al. Clinical, laboratory, roentgenographic, and electrocardiographic findings in patients with acute pulmonary embolism and no pre-existing cardiac or pulmonary disease. Chest. 1991;100 (3):598.

17. Martin AI, Rao G. COVID-19: a potential risk factor for acute pulmonary embolism. Methodist Debakey Cardiovasc J. 2020;16:155-7.

18. Lorenzo C, Francesca B, Francesco P, Elena C, Luca S, Paolo S. Acute pulmonary embolism in COVID-19 related hypercoagulability. J Thromb Thrombolysis. 2020; 50:223-226

19. Danzi G B, Loffi M, Galeazzi G, Gherbesi E. Acute pulmonary embolism and COVID-19 pneumonia: a random association? Eur Heart J. 2020;14;41(19):1858. doi: 10.1093/eurheartj/ ehaa254.

20. Tang Ning, et al. Anticoagulant treatment is associated with decreased mortality in severe coronavirus disease 2019 patients with coagulopathy. J Thromb Haemost. 2020;18(5):1094-1099. doi: 10.1111/jth.14817.

21. Bikdeli B, Madhavan MV, Jimenez D, Chuich T, Dreyfus I, Driggin E, et al. COVID-19 and thrombotic or thromboembolic disease: implications for prevention, antithrombotic therapy, and follow-up: JACC state-of-the-art review. J Am Coll Cardiol. 2020;75(23):2950-73.

22. Rotzingera DC, Beigelman-Aubrya C, von Garnierb C, Qanadli SD. Letter to the Editors in Chief: Pulmonary embolism in patients with COVID19: Time to change the paradigm of computed tomography. Thromb Research. 2020;190:58-9. Disponible en: https://www.ncbi.nlm.nih.gov/ pmc/articles/PMC7151364/ pdf/main.pdf.

23. Chung M, Bernheim A, Mei X, Zhang N, Huang M, Zeng X, et al. CT imaging features of 2019 novel coronavirus (2019-nCoV). Radiology. 2020; Feb 04. doi: 10.1148/radiol.2020200230. [Epub]. 